\title{
ASSET ALLOCATION WITH REGIME-SWITCHING: DISCRETE-TIME CASE
}

\author{
BY
}

\author{
Ka Chun Cheung and Hailiang Yang
}

\begin{abstract}
In this paper, we study the optimal asset allocation problem under a discrete regime switching model. Under the short-selling and leveraging constraints, the existence and uniqueness of the optimal trading strategy are obtained. We also obtain some natural properties of the optimal strategy. In particular, we show that if there exists a stochastic dominance order relationship between the random returns at different regimes, then we can order the optimal proportions we should invest in such regimes.
\end{abstract}

\section{KEY Words}

Optimal trading strategy, Power utility function, Finite-state Markovian Chain, Bellman equation, Stochastic order.

\section{INTRODUCTION}

Portfolio selection problem is one of the key topics in finance. Markowitz's mean-variance model (see Markowitz (1959)) is a one period model of portfolio selection. Samuelson (1969) extended the work of Markowitz to a dynamic model and considered a discrete time consumption investment model with the objective of maximizing the overall expected consumption. He advocated a dynamic stochastic programming approach and succeeded in obtaining the optimal decision for a consumption investment model. Merton (1969) first used the stochastic optimal control method in continuous finance. He was able to obtain a closed form solution to the problem of optimal portfolio strategy under specific assumptions about asset returns and investor preferences. He showed that, under the assumptions of geometric Brownian motion for the stock returns and HARA utility, the optimal proportion invested in the risky asset portfolio is constant through time. Later, Grauer and Hakansson (1982, 1985) used a discrete time approach to determine optimal asset allocations. They updated the joint distribution of asset returns every period and were able to incorporate time variation in the return distribution. Their conclusion was that active re-balancing among the major asset classes can substantially improve 
investment performance. Cox and Huang (1989) and Pliska (1986) introduced the martingale technique to deal with the consumption portfolio problem in continuous time under uncertainty. For a fairly general class of utility functions, Cox and Huang showed that in order to prove the existence of optimal controls, it suffices to check whether the parameters of a system of stochastic differential equations, derived completely from the price system, satisfy a local Lipschitz and uniform growth condition. This approach takes care of the nonnegativity constraint on consumption in a simple and direct way.

Recently, people in finance and actuarial science have also started paying attention to regime switching models. Di Masi et al. (1994) considered the European options under the Black-Scholes formulation of the market in which the underlying economy switches among a finite number of states. Buffington and Elliott (2001) discussed the American options under this set-up. Hardy (2001) used monthly data from the Standard and Poor's 500, and the Toronto Stock Exchange 300 indices to fit a regime-switching lognormal model. The fit of the regime-switching model to the data was compared with other econometric models. Zariphopoulou (1992) considered an investment consumption model with regime switching. Zhang (2001) derived an optimal stock selling rule for a Markov-modulated Black-Scholes model. Yin and Zhou (2003) studied a discrete-time version of Markowitz's mean-variance portfolio selection problem, where the market parameters depend on a finite-state Markov chain. In Zhou and Yin (2003) considered a continuous-time version of the Markowitz meanvariance portfolio selection problem for a market consisting of one bank account and multiple stocks. The market parameters depend on the market mode that switches among a finite number of states.

In this paper, we consider a discrete market consisting of one bank account and one risky asset. We use the Markovian regime switching formulation to model the risky asset's return. This model can capture the feature of possible change of the distribution of the stock return if the economical or political environment changes. The rest of the paper is arranged as follows: Section 2 provides the formulation of our model; Section 3 introduces an auxiliary function that is useful in this paper; Section 4 obtains the existence and uniqueness of the optimal strategy; Section 5 investigates some properties of the solution; Section 6 concludes the paper with some remarks.

\section{Model Formulation}

We will study the optimal asset allocation problem in a discrete-time, finite-horizon setting. We assume that there are only two assets being traded in the market: a risk-free bond and a risky stock. The risk-free bond will earn a deterministic return $R$ over a single time period (say, one year). The random return of the risky stock over different time periods will depend on the state of a finitestate Markov Chain $\left\{\xi_{n}\right\}$ at the beginning of that time period. The wealth of an investor at time $n$ will be denoted as $W_{n}$, and the random return in time period $[n, n+1]$ given $\xi_{n}=i$ as $R_{n}^{i}$. An investor at time $n$ has to decide the proportion of his/her wealth to be invested in the risky stock, and this proportion 
is denoted as $\alpha_{n}$. We will constrain this proportion to lie in the unit interval $[0,1]$, which means that short-selling and leveraging are prohibited. This restriction is to avoid the possibility of the wealth becoming negative. The wealth will evolve as

$$
W_{n}=W_{n-1}\left[\alpha_{n-1} R_{n-1}^{\xi_{n-1}}+\left(1-\alpha_{n-1}\right) R\right] \quad n=1,2, \ldots, N,
$$

where $N$ is the investment horizon (number of investment periods). Given that the initial wealth is $W_{0}$, and the initial state of the regime is $\xi_{0}$, the objective of the investor is to maximize the expected power-utility of the terminal wealth $W_{N}$ :

$$
\max _{\left\{\alpha_{0}, \alpha_{1}, \ldots, \alpha_{N-1}\right\} \subseteq[0,1]^{N}} \mathbb{E}_{0}\left[\frac{1}{\gamma} W_{N}^{\prime \prime}\right]
$$

over all the admissible investment strategies $\left\{\alpha_{0}, \ldots, \alpha_{N-1}\right\}$, where $\gamma$ is any constant that is strictly less than one but not equal to zero: $\gamma \in(-\infty, 0) \cup(0,1)$, and $\mathbb{E}_{0}$ is the expectation operator given the information at time 0 , i.e. given $\left(\xi_{0}, W_{0}\right)$. Specifically, an admissible investment strategy $\left\{\alpha_{0}, \ldots, \alpha_{N-1}\right\}$ is a feedback policy in which each $\alpha_{n}$ maps the wealth $W_{n}$ and regime $\xi_{n}$ at time $n$ into the decision variable $\alpha_{n}=\alpha_{n}\left(W_{n}, \xi_{n}\right) \in[0,1]$. The optimal feedback policy that maximizes the above expression is called the optimal trading strategy, and is denoted as $\left\{\hat{\alpha}_{0}, \hat{\alpha}_{1}, \ldots, \hat{\alpha}_{N-1}\right\}$.

We make the following assumptions throughout this paper:

1. $\left\{\xi_{n}\right\}$ is a discrete-time and time-homogeneous Markov Chain with state space $\mathcal{S}=\{1,2, \ldots, S\}$ and transition probability matrix $\mathrm{P}=\left(p_{i j}\right)$;

2. for fixed $i \in \mathcal{S}$, the random returns $R_{0}^{i}, R_{1}^{i}, \ldots, R_{N-1}^{i}$ are identically distributed, with distribution function $F_{i}$; they are assumed to be strictly positive and integrable;

3. the single-period return of the risk-free bond $R$ is a deterministic constant that is strictly greater than 1 ;

4. in different time periods, the random returns are independent, i.e. $\forall i, j \in \mathcal{S}$, $R_{n}^{i}$ is independent to $R_{m}^{j}$ for $m \neq n$;

5. the Markov Chain $\{\xi\}$ is independent to the random returns in the following sense:

$\mathbb{P}_{n}\left(\xi_{n+1}=j, R_{n}^{\xi_{n}} \in B\right)=\mathbb{P}_{n}\left(\xi_{n+1}=j\right) \mathbb{P}_{n}\left(R_{n}^{\xi_{n}} \in B\right)$

for all $j \in \mathcal{S}, B \in \mathfrak{B}(\mathbb{R})$ and $n=0,1, \ldots, N-1$, where $\mathbb{P}_{n}$ is the probability given the information up to time $n$.

\section{An AuXiLiary FunCtion}

The following auxiliary function turns out to be useful in studying our dynamic maximization problem. 
Definition 1. Fix any $i \in \mathcal{S}$, and assume that $R^{i}$ is a random variable with distribution function $F_{i}$. Define function $\widetilde{Q}_{i}(\cdot):[0,1] \longrightarrow \mathbb{R}$ by

$$
\widetilde{Q_{i}}(\alpha)=\mathbb{E}\left[\left(\alpha R^{i}+(1-\alpha) R\right)^{\gamma}\right] .
$$

The next proposition summarizes some basic properties of the function $\widetilde{Q}_{i}$. Further properties will be explored later.

Proposition 1. For fixed $i \in \mathcal{S}$, the function $\widetilde{Q}_{i}(\cdot)$ is

1. well-defined on $[0,1]$ when $0<\gamma<1$, i.e. for any $\alpha \in[0,1]$, the random variable $\left(\alpha R^{i}+(1-\alpha) R\right)^{\gamma}$ is integrable; when $\gamma<0$, it is well-defined if we impose the extra condition that $\left(R^{i}\right)^{y}$ is integrable;

2. non-negative on $[0,1]$;

3. strictly concave (strictly convex resp.) on $[0,1]$ when $0<\gamma<1(\gamma<0$ resp. $)$;

4. continuous on $[0,1]$.

Proof (1): We prove the case of $0<\gamma<1$ first. For any $0 \leq \alpha \leq 1$, note that $0<$ $\left(\alpha R^{i}+(1-\alpha) R\right)^{\gamma} \leq\left(R^{i}+R\right)^{\gamma} \leq \max \left\{1, R^{i}+R\right\} \leq 1+R^{i}+R$, which is integrable by our assumption that $R^{i}$ is integrable. For $\gamma<0$, we have $0<\left(\alpha R^{i}+(1-\alpha) R\right)^{\gamma} \leq$ $\left(\min \left\{R^{i}, R\right\}\right)^{\gamma}=\max \left\{\left(R^{i}\right)^{\gamma}, R^{\gamma}\right\} \leq\left(R^{i}\right)^{\gamma}+R^{\gamma}$, which is integrable under the extra assumption that $\left(R^{i}\right)^{\gamma}$ is integrable.

(2): Obvious.

(3): When $0<\gamma<1$, choose arbitrarily $\alpha_{1}, \alpha_{2} \in[0,1]$ with $\alpha_{1} \neq \alpha_{2}$, and $0<\beta<1$, we have

$$
\begin{aligned}
& \widetilde{Q_{i}}\left(\beta \alpha_{1}+(1-\beta) \alpha_{2}\right) \\
= & \mathbb{E}\left[\left\{\left(\beta \alpha_{1}+(1-\beta) \alpha_{2}\right) R^{i}+\left(1-\left(\beta \alpha_{1}+(1-\beta) \alpha_{2}\right)\right) \mathrm{R}\right\}^{\gamma}\right] \\
= & \mathbb{E}\left[\left\{\left(\beta \alpha_{1}+(1-\beta) \alpha_{2}\right)\left(R^{i}-R\right)+R\right\}^{\gamma}\right] \\
= & \mathbb{E}\left[\left\{\beta\left(\alpha_{1}\left(R^{i}-R\right)+R\right)+(1-\beta)\left(\alpha_{2}\left(R^{i}-R\right)+R\right)^{\gamma}\right]\right. \\
> & \mathbb{E}\left[\beta\left\{\alpha_{1}\left(R^{i}-R\right)+R\right\}^{\gamma}+(1-\beta)\left\{\alpha_{2}\left(R^{i}-R\right)+R\right\}^{\gamma}\right] \\
= & \beta \widetilde{Q_{i}}\left(\alpha_{1}\right)+(1-\beta) \widetilde{Q}_{i}\left(\alpha_{2}\right),
\end{aligned}
$$

where the inequality follows from the strict concavity of the function $x^{\gamma}$ on $(0, \infty)$. This shows the strict concavity of $\widetilde{Q}_{i}(\cdot)$ when $0<\gamma<1$. When $\gamma<0$, the function $x^{\gamma}$ is strictly convex on $(0, \infty)$, hence the ">" sign becomes " $<$ " in the above equation, which is the required strict convexity of $\widetilde{Q_{i}}(\cdot)$.

(4): From the proof of Part (1), we know that the collection of random variables $\left\{\left(\alpha R^{i}+(1-\alpha) R\right)^{\gamma}\right\}_{0 \leq \alpha \leq 1}$ is dominated by an integrable random variable. Continuity follows from the Dominated Convergence Theorem.

By the above proposition, we will hereafter make the extra assumption that $\left(\mathrm{R}^{i}\right)^{\gamma}$ is integrable whenever $\gamma$ is negative. As a consequence of Proposition $1, \widetilde{Q}_{i}(\cdot)$ achieves its maximum (minimum resp.) on [0,1] at a unique point when $0<\gamma$ $<1(\gamma<0$ resp. $)$, which will be denoted as $\alpha_{i}^{*}$. The corresponding maximum (minimum resp.) value $\widetilde{Q_{i}}\left(\alpha_{i}^{*}\right)$ is denoted as $Q_{i}^{(1)}$. Note that $Q_{i}^{(1)}$ is non-negative. 


\section{Optimal Trading Strategy}

Now we can return to our problem. In order to employ the dynamic programming technique, it is necessary to introduce the following concept:

Definition 2. The value function $V_{n}\left(\xi_{n}, W_{n}\right)$ is defined as

$$
V_{n}\left(\xi_{n}, W_{n}\right)=\max \mathbb{E}_{n}\left[\frac{1}{\gamma} W_{N}^{\prime}\right]=\max \mathbb{E}_{\xi_{n}, W_{n}}\left[\frac{1}{\gamma} W_{N}^{\prime}\right],
$$

where the maximum is performed over all the admissible investment strategies $\left\{\alpha_{n}\right.$, $\left.\alpha_{n+1}, \ldots, \alpha_{N-1}\right\}$.

Our objective is thus to compute $V_{0}\left(\xi_{0}, W_{0}\right)$ and find the associated optimal investment strategy. By the theory of Dynamic Programming, we know that the optimal trading strategy can be obtained by solving the following recursive equation, known as Bellman's Equation:

$$
\left\{\begin{array}{l}
V_{N}\left(\xi_{N}, W_{N}\right)=\frac{1}{\gamma} W_{N}^{\gamma} \\
V_{n}\left(\xi_{n}, W_{n}\right)=\max _{0 \leq \alpha_{n} \leq 1} \mathbb{E}_{n}\left[V_{n+1}\left(\xi_{n+1}, W_{n+1}\right)\right] \quad n=0,1, \ldots, N-1
\end{array}\right.
$$

Theorem 1. For $n=0,1,2, \ldots, N$, the value functions are given by

$$
V_{N-n}\left(\xi_{N-n}, W_{N-n}\right)= \begin{cases}\frac{1}{\gamma} W_{N-n}^{\prime} Q_{\xi_{N-n}}^{(n)} & n=1,2, \ldots, N, \\ \frac{1}{\gamma} W_{N}^{\prime} & n=0,\end{cases}
$$

where functions $Q_{i}^{(\cdot)}$ are defined recursively through

$$
Q_{i}^{(k+1)}=Q_{i}^{(1)} \sum_{j=1}^{S} p_{i j} Q_{j}^{(k)} \quad k=1,2, \ldots, N-1
$$

for any $i \in S$.

The optimal trading strategy, which solves Bellman's Equation, is given by

$$
\hat{\alpha}_{N-n}= \begin{cases}\alpha_{\xi_{N-n}}^{*} & n=1,2, \ldots, N, \\ 1 & n=0\end{cases}
$$

By Theorem 1, we can see that the optimal proportion of the wealth to be invested in the risky stock in a certain time period will depend on the regime at the beginning of that time period. However, it does not depend on the time remains. This is because the current decision will not affect the transition probability of the regime, nor will it affect the future random returns. 
Proof of Theorem 1: Obviously, equations (2) and (4) are true for $n=0$. We first show that (2) and (4) are true for $n$ equals 1, and then proceed by induction.

For $n=1$ and $0<\gamma<1$, we have

$$
\begin{aligned}
V_{N-1}\left(\xi_{N-1}, W_{N-1}\right)= & \max _{0 \leq \alpha_{N-1} \leq 1} \mathbb{E}_{N-1}\left[V_{N}\left(\xi_{N}, W_{N}\right)\right] \\
& =\max _{0 \leq \alpha_{N-1} \leq 1} \mathbb{E}_{N-1}\left[\frac{1}{\gamma} W_{N}^{\prime}\right] \\
& =\frac{W_{N-1}^{\prime}}{\gamma} \max _{0 \leq \alpha_{N-1} \leq 1} \mathbb{E}_{N-1}\left[\left(\alpha_{N-1} R_{N-1}^{\xi_{N-1}}+\left(1-\alpha_{N-1}\right) R\right)^{\gamma}\right] \\
& =\frac{W_{N-1}^{\prime}}{\gamma} \max _{0 \leq \alpha_{N-1} \leq 1} \tilde{Q}_{\xi_{N-1}}\left(\alpha_{N-1}\right) \\
& =\frac{W_{N-1}^{\prime}}{\gamma} \tilde{Q}_{\xi_{N-1}}\left(\alpha_{\xi_{N-1}}^{*}\right) \\
& =\frac{W_{N-1}^{\prime}}{\gamma} Q_{\xi_{N-1}}^{(1)} .
\end{aligned}
$$

When $\gamma<0$, the argument is exactly the same as above, except that in the third and the fourth lines, "max" is replaced by "min" because $\gamma$ is a negative number.

Now suppose that (2) and (4) are true for some $k \in\{1,2, \ldots, N-1\}$, then for $0<\gamma<1$ we have

$$
\begin{aligned}
& V_{N-(k+1)}\left(\xi_{N-(k+1)}, W_{N-(k+1)}\right) \\
& =\max _{0 \leq \alpha_{N-(k+1)} \leq 1} \mathbb{E}_{N-(k+1)}\left[V_{N-k}\left(\xi_{N-k}, W_{N-k}\right)\right] \\
& =\max _{0 \leq \alpha_{N-(k+1)} \leq 1} \mathbb{E}_{N-(k+1)}\left[\frac{1}{\gamma} W_{N-k}^{\prime} Q_{\xi_{N-k}}^{(k)}\right] \\
& =\frac{1}{\gamma} W_{N-(k+1)}^{\gamma} \mathbb{E}_{N-(k+1)}\left[Q_{\xi_{N-k}^{(k)}}^{(k)}\right] \\
& 0 \leq \alpha_{N-(k+1)} \leq 1 \\
& =\frac{1}{\gamma} W_{N-(k+1)}^{\gamma}\left\{\sum_{j=1}^{S} p_{\xi_{N-(k+1)}, j} Q_{j}^{(k)}\right\} \max _{0 \leq \alpha_{N-(k+1)} \leq \tilde{Q}_{\xi_{N-(k+1)}}\left(\alpha_{N-(k+1)}\right)}\left[\left(\alpha_{N-(k+1)} R_{N-(k+1)}^{\xi_{N-(k+1)}}+\left(1-\alpha_{N-(k+1)}\right) R\right)^{\gamma}\right] \\
& =\frac{1}{\gamma} W_{N-(k+1)}^{V}\left\{\sum_{j=1}^{S} p_{\xi_{N-(k+1)}, j} Q_{j}^{(k)}\right\} \tilde{Q}_{\xi_{N-(k+1)}}\left(\alpha_{\xi_{N-(k+1)}^{*}}^{*}\right) \\
& =\frac{1}{\gamma} W_{N-(k+1)}^{\gamma}\left\{\sum_{j=1}^{S} p_{\xi_{N-(k+1)}, j} Q_{j}^{(k)}\right\} Q_{\xi_{N-(k+1)}^{(1)}} \\
& =\frac{1}{\gamma} W_{N-(k+1)}^{\gamma} Q_{\xi_{N-(k+1)}}^{(k+1)} .
\end{aligned}
$$


Again, when $\gamma<0$, the "max" in the fifth and the sixth lines are replaced by "min". This shows that (2) and (4) are also true for $n$ equals $k+1$. This completes the induction step.

\section{Properties of the Solution}

We can see that the solution to our utility maximization problem is intimately related to the functions $Q_{i}^{(\cdot)}$ and its maximizer (or minimizer, depending on the sign of $\gamma) \alpha_{i}^{*}$. We will therefore study their properties carefully in this section. In particular, we will give a simple sufficient condition that will allow us to rank the $\alpha_{(\cdot)}^{*}$ 's.

Proposition 2. For fixed $i \in \mathcal{S}$, we have, when $0<\gamma<1$

$$
Q_{i}^{(1)} R^{-\gamma} \leq Q_{i}^{(2)} R^{-2 \gamma} \leq \ldots \leq Q_{i}^{(N)} R^{-N \gamma} .
$$

Similarly, when $\gamma<0$, we have

$$
Q_{i}^{(1)} R^{-\gamma} \geq Q_{i}^{(2)} R^{-2 \gamma} \geq \ldots \geq Q_{i}^{(N)} R^{-N \gamma} .
$$

Proof: We will prove equation (5) first. Note that, for any $i \in \mathcal{S}$, we have the simple inequality:

$$
Q_{i}^{(1)}=\max _{0 \leq \alpha \leq 1} \mathbb{E}\left[\left(\alpha R^{i}+(1-\alpha) R\right)^{\gamma}\right] \geq \mathbb{E}\left[R^{\gamma}\right]=R^{\gamma} .
$$

From this, we then have

$$
Q_{i}^{(2)} R^{-2 \gamma}=Q_{i}^{(1)}\left[\sum_{j=1}^{S} p_{i j} Q_{j}^{(1)}\right] R^{-2 \gamma} \geq Q_{i}^{(1)}\left[\sum_{j=1}^{S} p_{i j}\left(R^{\gamma}\right)\right] R^{-2 \gamma}=Q_{i}^{(1)} R^{-\gamma} .
$$

Assume that for some $2 \leq k \leq N-1, Q_{i}^{(k)} R^{-k \gamma} \geq Q_{i}^{(k-1)} R^{-(k-1) \gamma}$ for all $i \in \mathcal{S}$. Then

$$
\begin{aligned}
Q_{i}^{(k+1)} R^{-(k+1) \gamma} & =Q_{i}^{(1)}\left[\sum_{j=1}^{S} p_{i j} Q_{j}^{(k)}\right] R^{-(k+1) \gamma} \\
& \geq Q_{i}^{(1)}\left[\sum_{j=1}^{S} p_{i j}\left(Q_{j}^{(k-1)} R^{\gamma}\right)\right] R^{-(k+1) \gamma} \\
& =Q_{i}^{(1)}\left[\sum_{j=1}^{S} p_{i j} Q_{j}^{(k-1)}\right] R^{-k \gamma} \\
& =Q_{i}^{(k)} R^{-k \gamma} .
\end{aligned}
$$

This completes the induction step and finishes the proof of equation (5). 
For the case where $\gamma<0$, we have the following instead of equation (7):

$$
Q_{i}^{(1)}=\min _{0 \leq \alpha \leq 1} \mathbb{E}\left[\left(\alpha R^{i}+(1-\alpha) R\right)^{\gamma}\right] \leq \mathbb{E}\left[R^{\gamma}\right]=R^{\gamma} .
$$

The proof is exactly the same as above, except that the " $\geq$ " signs in the inequalities are replaced by " $\leq$ " signs.

From this proposition and its proof, it directly follows that for $i \in \mathcal{S}$ :

- when $0<\gamma<1$,

$$
\left\{\begin{array}{l}
Q_{i}^{(1)} \leq Q_{i}^{(2)} \leq \ldots \leq Q_{i}^{(N)}, \\
Q_{i}^{(n)} \geq R^{n \eta}
\end{array}\right.
$$

- when $\gamma<0$,

$$
\left\{\begin{array}{l}
Q_{i}^{(1)} \geq Q_{i}^{(2)} \geq \ldots \geq Q_{i}^{(N)}, \\
Q_{i}^{(n)} \leq R^{n y}
\end{array}\right.
$$

From Theorem 1, we know that if $N$ is the investment horizon of the investor, then the maximum expected discounted utility (using $R^{-1}$ as the single period discount rate) is given by $\frac{\mathscr{W}^{\prime \prime}}{\gamma} Q_{\xi_{0}}^{(N)} R^{-N y}$. Therefore, Proposition 2 implies that as long as the initial wealth and the initial regime remain unchanged, then the expected discounted utility will increase as investment horizon lengthens.

Given any two random variables $X$ and $Y$, we say that $X$ is larger than $Y$ in the sense of second order stochastic dominance, denoted by $Y \leq_{\mathrm{SSD}} X$, if

$$
\mathbb{E}[f(Y)] \leq \mathbb{E}[f(X)]
$$

for any increasing concave function $f$ provided that the expectations exist. Similarly, if distribution functions of $X$ and $Y$ are denoted by $F(x)$ and $G(x)$, respectively, and $Y \leq_{\text {SSD }} X$, we say that $G \leq_{\text {SSD }} F$. It can be seen that this order is a partial order on the set of all random variables. $(X=Y$ means that $X \stackrel{d}{=} Y)$. For detailed discussions on stochastic orders and their relationships, we refer the readers to Shaked and Shanthikumar (1994).

Proposition 3. Let $i, j$ be in S. If $F_{j} \leq_{\mathrm{SSD}} F_{i}$, then when $0<\gamma<1(\gamma<0$ resp.)

$$
Q_{j}^{(1)} \leq(\geq) Q_{i}^{(1)} .
$$

The intuitive meaning of the condition $F_{j} \leq_{\mathrm{SSD}} F_{i}$ is that random investment return in regime $i$ is more favorable than that in regime $j$ to a risk-averse investor 
who have an increasing concave utility function. Regime $i$ thus represents a more favorable investment environment than regime $j$. This proposition says that, in a "better" investment environment, an investor can achieve a higher expected utility over a single period.

Proof of Proposition 3: Suppose $F_{j} \leq_{\mathrm{SSD}} F_{i}$. First, we note that

$$
\widetilde{Q_{i}}(0)=\mathbb{E}\left[R^{\gamma}\right]=\widetilde{Q_{j}}(0),
$$

and for any fixed $0<\alpha \leq 1$, the function $f(x)=[\alpha x+(1-\alpha) R]^{\gamma}$ is increasing and concave (decreasing and convex resp.) when $0<\gamma<1(\gamma<0$ resp.). By the definition of stochastic domination, we have

$$
\begin{array}{rll}
\widetilde{Q}_{i}(\alpha) & = & \mathbb{E}\left[\left(\alpha R^{i}+(1-\alpha) R\right)^{\gamma}\right] \\
& = & \mathbb{E}\left[f\left(R^{i}\right)\right] \\
\geq(\leq) & & \mathbb{E}\left[f\left(R^{j}\right)\right] \\
& = & \mathbb{E}\left[\left(\alpha R^{j}+(1-\alpha) R\right)^{\gamma}\right] \\
& = & \widetilde{Q_{j}}(\alpha) .
\end{array}
$$

Combining equations (11) and (12), we have shown that $\widetilde{Q_{j}}(\alpha) \leq(\geq) \widetilde{Q}_{i}(\alpha)$ for all $\alpha \in[0,1]$. Thus, we have $Q_{i}^{(1)}=\widetilde{Q}_{i}\left(\alpha_{i}^{*}\right) \geq(\leq) \widetilde{Q_{i}}\left(\alpha_{j}^{*}\right) \geq(\leq) \widetilde{Q_{j}}\left(\alpha_{j}^{*}\right)=Q_{j}^{(1)}$.

Note that, in general, the condition $F_{j} \leq_{\mathrm{SSD}} F_{i}$ is not sufficient to guarantee that $Q_{j}^{(n)} \leq Q_{i}^{(n)}$ (or $Q_{j}^{(n)} \geq Q_{i}^{(n)}$, depending on the sign of $\gamma$ ) for $\mathrm{n}>1$. The structure of the transition matrix $P$ also plays a significant role. Given any $m \times m$ matrix $\mathrm{A}=\left(a_{i j}\right)$, supposing that its elements satisfy $a_{i j} \geq 0$ and $\sum_{j} a_{i j}=1$, it is said to be stochastically monotone if

$$
\sum_{k \geq r} a_{j k} \leq \sum_{k \geq r} a_{i k}
$$

for all $1 \leq i, j, r \leq m$ with $j \leq i$. It is not difficult to prove the following lemma:

Lemma 1. Suppose $\mathrm{A}=\left(a_{i j}\right)$ is a $m \times m$ matrix, $a_{i j} \geq 0$ and $\sum_{j} a_{i j}=1$, and assume that $\mathrm{A}$ is stochastically monotone. Then, for any increasing (decreasing resp.) column vector $\mathrm{c}=\left(c_{1}, c_{2}, \ldots, c_{m}\right)$, the column vector $\mathrm{Ac}$ is also increasing (decreasing resp.).

For more details, see for example Rolski et al. (1999). The following proposition gives a sufficient condition to allows us to rank $Q_{1}^{(n)}, \ldots, Q_{S}^{(n)}$.

Proposition 4. Suppose for any $i, j \in \mathcal{S}$ with $i \neq j$, we have either $F_{j} \leq_{\mathrm{SSD}} F_{i}$ or $F_{i} \leq_{\mathrm{SSD}} F_{j}$. By the transitivity of the stochastic dominance order, we may assume without loss of generality that

$$
F_{1} \leq_{\mathrm{SSD}} F_{2} \leq_{\mathrm{SSD}} F_{3} \leq_{\mathrm{SSD}} \cdots \leq_{\mathrm{SSD}} F_{S}
$$


If the transition matrix $\mathrm{P}$ is stochastically monotone, then whenever $n>1$ and $i, j \in \mathcal{S}$ with $i>j$, we have

$$
Q_{j}^{(n)} \leq(\geq) Q_{i}^{(n)}
$$

when $0<\gamma<1(\gamma<0$ resp. $)$.

Proof: We will only prove the case of $0<\gamma<1$, the case of $\gamma<0$ can be proved similarly. By Proposition 3 and (14), we have

$$
Q_{1}^{(1)} \leq Q_{2}^{(1)} \leq \cdots \leq Q_{S}^{(1)} .
$$

Suppose $Q_{1}^{(k)} \leq Q_{2}^{(k)} \leq \cdots \leq Q_{S}^{(k)}$ holds for $k=1,2, \ldots, N-1$. If P is stochastically monotone, then for any $j \leq i$, we have

$$
Q_{j}^{(k+1)}=Q_{j}^{(1)} \sum_{l=1}^{S} p_{j l} Q_{l}^{(k)} \leq Q_{j}^{(1)} \sum_{l=1}^{S} p_{i l} Q_{l}^{(k)} \leq Q_{i}^{(1)} \sum_{l=1}^{S} p_{i l} Q_{l}^{(k)}=Q_{i}^{(k+1)}
$$

in which the first inequality follows from Lemma 1.

As we have discussed before, the stochastic dominance condition $F_{j} \leq_{\mathrm{SSD}} F_{i}$ means that the investment environment in regime $i$ is better than that in regime $j$. It is natural to ask whether we should invest a larger proportion of our wealth into the risky stock. The following proposition gives an affirmative answer to this question when $0<\gamma<1$.

Proposition 5. Let $i, j$ be in $\mathcal{S}$. If $F_{j} \leq_{\mathrm{SSD}} F_{i}$, then when $0<\gamma<1$,

$$
\alpha_{j}^{*} \leq \alpha_{i}^{*} .
$$

We need the following Lemma in order to prove this Proposition.

Lemma 2: Let $i$ be in $S$, and $0<\gamma<1$.

(i) The function $\widetilde{Q}_{i}(\alpha)$ is differentiable with respect to $\alpha$ on the open interval $(0,1)$;

(ii) If the function $\widetilde{Q}_{i}(\alpha)$ achieves its maximum at $\alpha_{i}^{*} \in(0,1)$, then

$$
\begin{gathered}
\mathbb{E}\left[\left(\alpha_{i}^{*} R^{i}+\left(1-\alpha_{i}^{*}\right) R\right)^{\gamma}\right] \\
=R \mathbb{E}\left[\left(\alpha_{i}^{*} R^{i}+\left(1-\alpha_{i}^{*}\right) R\right)^{\gamma-1}\right] .
\end{gathered}
$$

Proof (i): Fix any $0<\varepsilon_{1}<\varepsilon_{2}<1$. Recall from the definition that $\widetilde{Q}_{i}(\alpha)=\mathbb{E}\left[\left(\alpha R^{i}\right.\right.$ $+(1-\alpha) R)^{\gamma}$ ], where $R^{i}$ has distribution $F_{i}$. The derivative of the expression inside the expectation with respect to $\alpha$ is given by

$$
\gamma\left(\alpha R^{i}+(1-\alpha) R\right)^{\gamma-1}\left(R^{i}-R\right) .
$$


For $\varepsilon_{1}<\alpha<\varepsilon_{2}$, observe the following:

$$
\begin{aligned}
\left|\frac{R^{i}-R}{\left(\alpha R^{i}+(1-\alpha) R\right)^{1-\gamma}}\right| & \leq \frac{\left|R^{i}-R\right|}{((1-\alpha) R)^{1-\gamma}} \\
& \leq \frac{\left|R^{i}\right|+|R|}{\left(\left(1-\varepsilon_{2}\right) R\right)^{1-\gamma}}
\end{aligned}
$$

where the last fraction is integrable by our assumption that $R^{i}$ is integrable. This implies that the collection of random variables

$$
\left\{\left(\alpha R^{i}+(1-\alpha) R\right)^{\gamma-1}\left(R^{i}-R\right)\right\}_{\varepsilon_{1}<\alpha<\varepsilon_{2}}
$$

is uniformly integrable, and hence $\widetilde{Q_{i}}(\alpha)$ is differentiable on the open interval $\left(\varepsilon_{1}, \varepsilon_{2}\right)$. As $\varepsilon_{1}$ and $\varepsilon_{2}$ are arbitrarily chosen on $(0,1)$, we conclude that $\widetilde{Q}_{i}(\alpha)$ is differentiable on $(0,1)$.

(ii): As $\widetilde{Q_{i}}(\alpha)$ is differentiable on $(0,1)$, we may denote the derivative as

$$
\left.\frac{d}{d \alpha} \tilde{Q}_{i}(\alpha)\right|_{a=h}=D_{i}(h)
$$

for $0<h<1$, which is equal to $\gamma \mathbb{E}\left[\left(h R^{i}+(1-h) R\right)^{\gamma-1}\left(R^{i}-R\right)\right]$. If the maximizer $\alpha_{i}^{*}$ lies in the open interval $(0,1)$, we have the first order necessary condition

$$
D_{i}\left(\alpha_{i}^{*}\right)=0,
$$

which means

$$
\gamma \mathbb{E}\left[\left(\alpha_{i}^{*} R^{i}+\left(1-\alpha_{i}^{*}\right) R\right)^{\gamma-1}\left(R^{i}-R\right)\right]=0 .
$$

By multiplying both sides by $\frac{a_{i}^{*}}{\gamma}$, then adding the term $R \mathbb{E}\left[\left(\alpha_{i}^{*} R^{i}+\left(1-\alpha_{i}^{*}\right) R\right)^{\gamma-1}\right]$ to both sides, we can obtain (17).

Now we can return to the proof of Proposition 5.

Proof of Proposition 5: In order to prove that $\alpha_{j}^{*} \leq \alpha_{i}^{*}$, we consider the following three cases: (i) $\alpha_{j}^{*}=0$, (ii) $0<\alpha_{j}^{*}<1$, and (iii) $\alpha_{j}^{*}=1$. In fact, for case (i), (16) holds trivially.

For case (ii), because of the concavity of $\widetilde{Q}_{i}(\cdot)$, it is enough to prove that $D_{i}\left(\alpha_{j}^{*}\right)$ is non-negative. This can be proved as follows, using (12) and (17):

$$
\begin{aligned}
& D_{i}\left(\alpha_{j}^{*}\right) \\
= & \gamma \mathbb{E}\left[\left(\alpha_{j}^{*} R^{i}+\left(1-\alpha_{j}^{*}\right) R\right)^{\gamma-1}\left(R^{i}-R\right)\right]
\end{aligned}
$$




$$
\begin{aligned}
& =\frac{\gamma}{\alpha_{j}^{*}}\left\{\mathbb{E}\left[\left(\alpha_{j}^{*} R^{i}+\left(1-\alpha_{j}^{*}\right) R\right)^{\gamma}\right]-R \mathbb{E}\left[\left(\alpha_{j}^{*} R^{i}+\left(1-\alpha_{j}^{*}\right) R\right)^{\gamma-1}\right]\right\} \\
& \geq \frac{\gamma}{\alpha_{j}^{*}}\left\{\mathbb{E}\left[\left(\alpha_{j}^{*} R^{j}+\left(1-\alpha_{j}^{*}\right) R\right)^{\gamma}\right]-R \mathbb{E}\left[\left(\alpha_{j}^{*} R^{i}+\left(1-\alpha_{j}^{*}\right) R\right)^{\gamma-1}\right]\right\} \\
& =\frac{\gamma R}{\alpha_{j}^{*}}\left\{\mathbb{E}\left[\left(\alpha_{j}^{*} R^{j}+\left(1-\alpha_{j}^{*}\right) R\right)^{\gamma-1}\right]-\mathbb{E}\left[\left(\alpha_{j}^{*} R^{i}+\left(1-\alpha_{j}^{*}\right) R\right)^{\gamma-1}\right]\right\} \\
& \geq 0
\end{aligned}
$$

where the last inequality follows from (9) by noting that the function $f(x)=$ $-\left(\alpha_{j}^{*} x+\left(1-\alpha_{j}^{*}\right) R\right)^{\gamma-1}$ is increasing and concave on $[0, \infty)$.

For case (iii), due to the concavity of the function $\widetilde{Q}_{i}(\cdot)$ on the interval $[0,1]$, we have the following relationship:

$$
D_{i}(1) \geq 0 \Leftrightarrow \alpha_{i}^{*}=1 \quad \text { for } i \in \mathcal{S} .
$$

Thus, $D_{j}(1)$ is non-negative if $\alpha_{j}^{*}=1$. Define function $h(x)=\gamma\left(x^{\gamma}-R x^{\gamma-1}\right)$ on $(0, \infty)$. It is an increasing concave function, and $\mathbb{E}\left[h\left(R^{i}\right)\right]=D_{i}(1)$. The condition $F_{j} \leq_{\mathrm{SSD}} F_{i}$ then implies

$$
D_{i}(1)=\mathbb{E}\left[h\left(R^{i}\right)\right] \geq \mathbb{E}\left[h\left(R^{j}\right)\right]=D_{j}(1) \geq 0 .
$$

Thus $D_{i}(1)$ is non-negative. By (19) again, we have $\alpha_{i}^{*}=1$.

\section{CONCLUSION}

In this paper, we have studied a multi-period utility maximization problem, in which an investor can invest in either a risk-free bond or a risky stock. In different time periods, the return distributions of the risky stock are different, and depend on the current regime. Different regimes may be interpreted as different investment environments, and the switching between different regimes is modeled by a time-homogeneous Markov Chain. Under the short-selling and leveraging constraints, we have shown the existence and uniqueness of the optimal trading strategy. Various properties of the solution have been examined, and a sufficient condition to rank the optimal proportions of wealth invested in the risky stock under different regimes has been given.

\section{ACKNOWLEDGMENTS}

The authors would like to thank the referee for helpful suggestions and comments. The work described in this paper was supported by a grant from the Research Grants Council of the Hong Kong Special Administrative Region, China (Project No. HKU 7139/01H). 


\section{REFERENCES}

Buffington, J. and Elliott, R.J. (2001) American Options with Regime Switching, QMF Conference 2001, Sydney, Australia.

Cox, J.C. and Huang, C.F. (1989) Optimal consumption and Portfolio Policies when asset prices follow a diffusion process, Journal of Economic Theory, 49, 33-83.

Di Masi, G.B., Kabanov, Y.M. and Runggaldier, W.J. (1994) Mean Variance Hedging of Options on Stocks with Markov Volatility, Theory of Probability and Applications, 39, 173-181.

GraUeR, R.R. and HaKansson, N.H. (1982) Higher return, lower risk: historical returns on long run actively managed portfolios of stocks bonds and bills: 1936-1978, Financial Analysts Journal, March-April.

GRAUER, R.R. and HAKANSSON, N.H. (1985) Returns on levered, actively managed long run portfolios of stocks, bonds and bills: 1934-1983, Financial Analysts Journal, September-October.

Hardy, M.R. (2001) A Regime-Switching Model of Long-Term Stock Returns, North American Actuarial Journal, 5(2), 41-53.

Markowitz, H. (1959) Portfolio Selection: Efficient Diversification of Investment, New York. John Wiley and Sons.

Merton, R.C. (1969) Lifetime portfolio selection under uncertainty: The continuous-time case, Review of Economics and Statistics, 51, 247-257.

PlisKA, S.R. (1986) A Stochastic Calculus Model of Continuous Trading: Optimal Portfolios, Math. Oper. Res., 11, 371-382.

Rolski, T., Schmidli, H., Schmidt, V. and Teugels, J. (1999), Stochastic Processes for Insurance and Finance, John Wiley and Sons.

SAmuelson, P.A. (1969) Lifetime Portfolio Selection by Dynamic Stochastic Programming, The Review of Economics and Statistics, 51, 239-246.

Shaked, M. and Shanthikumar, J.G. (1994) Stochastic Orders and Their Applications, Academic Press.

YIN, G. and ZHou, X.Y. (2003) Markowitz's mean-variance portfolio selection with regime switching: From discrete-time models to their continuous-time limits, IEEE Transactions on Automatic Control, To appear.

ZARIPHOPOUlOU, T. (1992) Investment-consumption models with transactions costs and Markovchain parameters, SIAM J. Control Optim., 30, 613-636.

ZHANG, Q. (2001) Stock trading: An optimal selling rule, SIAM J. Control Optim., 40, 64-87.

ZHou, X.Y. and YIN, G. (2003) Markowitz mean-variance portfolio selection with regime switching: A continuous-time model, SIAM J. Control Optim., To appear.

Ka Chun Cheung and Hailiang Yang

Department of Statistics and Actuarial Science

The University of Hong Kong

Pokfulam Road, Hong Kong 